\title{
High-reliability Vehicle Detection and Lane Collision Warning System
}

\author{
Yassin Kortli $^{\mathrm{a}}$, Mehrez Marzougui ${ }^{\mathrm{b}}$, and Mohamed Atri ${ }^{\mathrm{a}}$ \\ ${ }^{a}$ Electronic and Micro-electronic Laboratory, Faculty of Sciences of Monastir, University of Monastir \\ ${ }^{b}$ Department of Computer Engineering, King Khalid University
}

Received: 19 January 2018; Accepted: 13 February 2018; Published: 08 March 2018

\begin{abstract}
In the last two decades, developing Driving Assistance Systems for security has been one of the most active research fields in order to minimize traffic accidents. Vehicle detection is a vital operation in most of these applications. In this paper, we present a high reliable and real-time lighting-invariant lane collision warning system. We implement a novel real-time vehicles detection using Histogram of Oriented Gradient and Support Vector Machine which could be used for collision prediction. Thus, in order to meet the conditions of real-time systems and to reduce the searching region, Otsu's threshold method play a critical role to extract the Region of Interest using the gradient information firstly. Secondly, we use Histogram of Oriented Gradient (HOG) descriptor to get the features vector, and these features are classified using a Support Vector Machine (SVM) classifier to get training base. Finally, we use this base to detect the vehicles in the road. Two sets generated the training data of our system a set of negative images (non-vehicles) a set of positive images (vehicles), and the test is performed on video sequences on the road. The proposed methodology is tested in different conditions. Our experimental results and accuracy evaluation indicates the efficiency of your system proposed for vehicles detection.
\end{abstract}

Index Terms: Driving Assistance Systems (DAS); Front Collision Warning System (FCWS); Otsu threshold; Histogram of Oriented Gradient (HOG); Support Vector Machine (SVM).

(C) 2018 Published by MECS Publisher. Selection and/or peer review under responsibility of the Research Association of Modern Education and Computer Science

\section{Introduction}

Driving Assistance Systems (DAS) such as front collision and lane departure warning has increases safe and

* Corresponding author. Yassin Kortli

E-mail address: yassin.kortli@isen-ouest.yncrea.fr 
secure driving. This system used to adjust, enhance, and automate the driving. The majority of traffic accidents happen because of drivers lack attention. Driving Assistance Systems reduces the driver workload and provides security. The system either alerts the driver whenever a dangerous situation is encountered. This, this paper focus on implementing Front Collision Warning System (FCWS) in realtime. The FCWS is an important module in research and development of Intelligent Transportation Systems (ITS). FCWS based on monocular vision, see itself as a key to avoiding deaths by accident with high reliability and low cost. Different systems have been implemented in the literature in order to detect the vehicles in front and enhance the security .on the road. All of these techniques can be evaluated using two separate phases: the learning phase and the detection phase. Moreover, an FCWS present three major processing modules [1]:

- $\quad$ Preprocessing.

- Feature extraction to store the features extracted from datasets (vehicles and no vehicles).

- Detection by matching the features stored in the system datasets with the features extracted from the vehicles testing in the videos sequences.

Many algorithms have been implemented in order to detect faces. Viola and Jones [7] implemented VIOLA JONES descriptor is used for face detection. This approach is done with tow techniques; the Haar features used to extract the features vector of the faces and Adaboost classifier for detection faces. But, this approach presents high computational complexities and does not solve the illumination problem. Local Binary Patterns, SURF, SIFT, and Gabor filter [3] are some of many other techniques quoted in the literature. However, the biggest problem of these techniques is unsatisfactory performance and high computational complexities under various lighting conditions. In this work, we propose to combine the use of Histogram Of Oriented Gradient (HOG) descriptor [9] with SVM classifier for vehicle detection. Histogram Of Oriented Gradient (HOG) descriptor is an adequate descriptor in terms of robustness and discrimination to extract "gradient distribution" vehicle features compared with the Haar descriptor [7]. As we explained bellow, this technique is considered since they can be used for learning and detection processes. In this research, the major contributions of our work can be summarized as follows:

- We applied the OTSU segmentation approach to segment the input frames and to cope with the lighting problem. This method is robust in the presence of illumination conditions, shadow, noise and lack of lane painting.

- According to the gradient information obtained by the OTSU segmentation method, we propose to determine a simple adaptive ROI by using a horizon line, aiming at reducing the computational complexity due to the processing time. Processing entire pixels of the full image is unnecessary.

- Histogram of Gradient (HOG) combined with SVM classifier is an adequate technique in terms of decreasing the false positive rate as well as reducing the amount of computation necessary to extract "gradient distribution" vehicle features and these features are then used to train with Support Vector Machine (SVM) classifier to get a training base.

- The training base is used to detect the vehicles in the road and, our system determines if there is traffic collision with the vehicle in front or not, depending on the car position.

This paper presents a novel vehicle detection system used on monocular vision under various lighting conditions in real time. Following the introduction, the rest of this paper is structured as follows: Section 2 explains the overview of the research related to vehicle detection. Section 3 describes a proposed vehicle detection system. Section 4 presents and discusses data collected through experiments under various lighting conditions. The last section, concludes the work and discusses future research directions. 


\section{Related Works}

In recent years, detecting a vehicle in real time using monocular vision has become a reputed area of studies in the intelligent vehicles community. From simpler image features such as symmetry and edges are used to detect vehicle rather. In the computer vision literature, these feature sets allow for detection and classification direct of vehicles in images. Two main classes are applied to identify vehicle: learning and detection phases. Learning phase consists an extract the training by the feature vector, and a classifier is trained to indicate the class of vehicles or not vehicles using image datasets. Detection phase after preprocessing step consists in obtained the feature vector for each image with the algorithm used in the learning phase. Finally, the presence of the vehicles or not in road scene detected by the same classifier. Gradient features or color features, and texture features are applied for learning and detection phase.

In [2] proposed a driving assistance system for detecting the first vehicle and extract the positions of the lane boundaries. For the front vehicle detection, vertical edges, symmetry properties, and features of undersides are used. In [3] implemented a Gabor filter for the front vehicle features extraction and a support vector machine (SVM) for classification. Due to selectivity in orientation, selectivity in scale and global illumination, Gabor filter gave an effect for getting a major degree of invariance to intensity. Vehicles do include the statistics of these features (e.g., skewness, standard deviation, and mean). Thus, high lines and edges at various scales and orientation could he very powerful for vehicle detection. They proposed Gabor filter to enhance filter banks, and deleted redundant filtering and used clustering to extract the filters that feature parameters that are similar. These features are then used to train an SVM classifier. Others are proposed motion classification [4], geometric features [5] [6] shadow detection [7] to detect vehicle and pedestrian using the monocular camera. Only the vehicles detection is implemented in this paper because there are almost cars in the road scenes.

Haar and Histogram of oriented gradient (HOG) gradient features are used in the object detection research, as they are in the vehicle detection research. To extract the features of HOG, we evaluate the edges operators and then ditching and discretizing the directions of the pixel intensities into a histogram [9], [1] and [6]. HOG features are presented excellent detection presentation and robust image features in a range of computer vision tasks. In [9], HOG method has been successfully implemented to detect, animals, pedestrian, and traffic sign. HOG detection strategy is presented one breakthrough in object detection occurs in a gradient feature domain. The major disadvantage of HOG features is that they are quite slow to calculate.

In Haar features are ideal for real-time detection of vehicle parts or vehicles because of the following benefits, such as horizontal and symmetrical structures, sensitive to vertical, and efficient to calculate [10] and [8]. In [8], presented a vehicle detection system. The Haar descriptor features of the image and the artificial neuron networks classifier to construct this system. The process of the integral image is used to represent the picture to enhance speed in the computation gets features by the descriptor. Two sets performed the learning of this system a set of negative images (non-vehicles) a set of positive images (vehicles), and the test is performed on another set of scenes (negative or positive). Also, color-based detection can be used to extract the object's edge using segmentation methods, especially in the day time. The segmentation methods are used to ignore unsuitable noise or regions, and to intensify certain colors. The extraction of a proper color space such as RGB, HSV, LUV or HIS is preferably headed in the segmentation methods[13,14,12].

However, existing methods still have high computational complexities and poor performances under different lighting conditions. For solving all the problems mentioned above, we proposed a very advanced and efficient system for vehicles detection, which works well under different lighting conditions.

\section{Proposed Method}

In this section, we describe our proposed system. For each image obtained by a monocular camera mounted on the front-view mirror, preprocessing step applied for in order to extract the ROI of the image. Also, in the preprocessing step, our proposed system is done in learning phase in order to form a training base using two 
sets of positive (vehicle) and negative (no vehicle) images in gray levels. After extract the ROI and getting the training base, we use this base in the detection process. Lastly, our system determines if there is traffic collision with the vehicle in front or not, depending on the car position. The flowchart of our proposed system is posted in Fig.1.

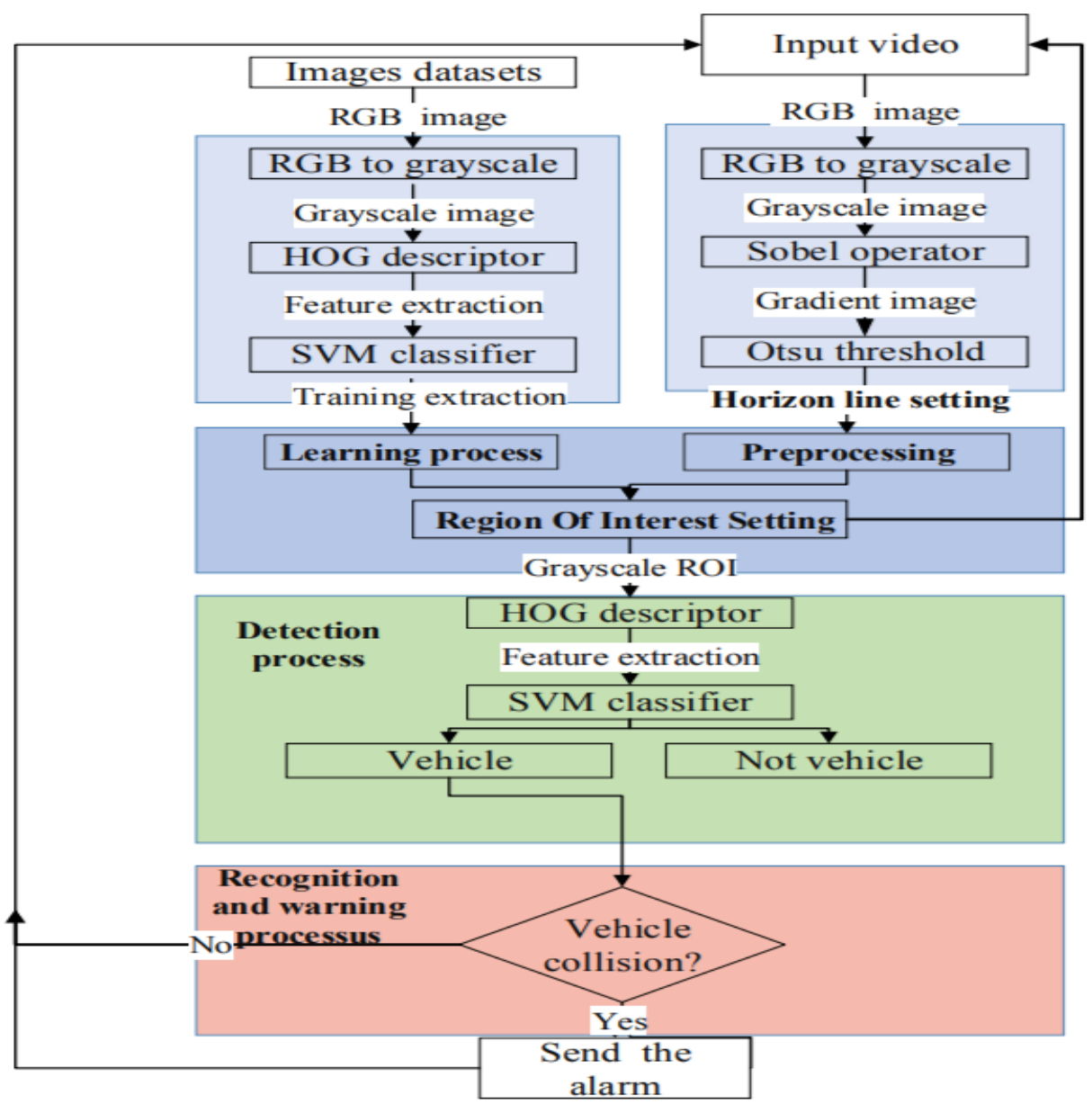

Fig.1. Proposed LCWS Flow Chart

\subsection{Preprocessing and Learning Processes}

In this section, we describe the preprocessing and learning processes of our proposed system. For each image obtained from the training datasets, preprocessing step is applied to enhance the features extraction in the image. Also, our proposed system is done by learning phase in order to form a training base.

\section{- Preprocessing}

A preprocessing step is an important aspect of the vehicles detection. The major goal of preprocessing is to detect the horizon line and extract the ROI for following steps of the input image. As displayed in Fig.2. the input color image is first converted to the grayscale image, followed. Next, a Sobel operator to detect the edges 
in the grayscale image. Then, in order to deal with lighting problem of the extraction of vehicles and gradient information, we use Otsu's method.

The color image (Fig.2.a) is first converted to the gray-scale image (Fig.2.b) as its treatment is minimal compared to a color image. This process transforms a 24-bit, color image to an 8-bit, grayscale image and also it affects the processing time. Thus, the conversion formula defined by Eq.1. [6] to take the value of the corresponding pixel in the grayscale image.

$$
\text { Grayscale }=0.299 * \mathrm{R}+0.587 * \mathrm{G}+0.114 * \mathrm{~B}
$$

After getting the grayscale image, the Sobel operator used for edge detection. The two Sobel operators used to detect the edge of the grayscale image can be described as:

$$
\mathrm{G}_{\mathrm{x}}=\left[\begin{array}{ccc}
-1 & -2 & -1 \\
0 & 0 & 0 \\
1 & 2 & 1
\end{array}\right], \mathrm{G}_{\mathrm{y}}=\left[\begin{array}{ccc}
-1 & 0 & 1 \\
-2 & 0 & 2 \\
-1 & 0 & 1
\end{array}\right]
$$

The gradient magnitude for each Sobel operator can be computed using Equation (2).

$$
g_{x}=I^{*} G_{x} \text { and } g_{y}=I^{\prime *} G_{y}
$$

with $\mathrm{I}^{\prime}$ is a grayscale image, $\mathrm{G}_{\mathrm{x}}$ and $\mathrm{G}_{\mathrm{y}}$ are the two Sobel operators and * denote the convolution operator. The gradient magnitude value $\mathrm{G}(\mathrm{x}, \mathrm{y})$ for each pixel $(\mathrm{x}, \mathrm{y})$ can be computed as follows:

$$
\mathrm{G}(\mathrm{x}, \mathrm{y})=\sqrt{g_{x}^{2}+g_{y}^{2}}
$$

After Sobel operator "gradient image", the image inevitably contains noise, caused by the lighting conditions different. Thus, we introduce Otsu's method to get the gradient information and deal with the lighting problem. A binary image get with Otsu's method means preserving researched information while removing unnecessary data to get better exact results. The key to a binary image is choosing an appropriate threshold, which is better to adaptive. According to Otsu's Method [11], the binary image was divided as two phases: background and foreground. Average value of the binary image could be expressed as:

$$
\mu(\mathrm{t})=\omega_{1}(\mathrm{t}) \mu_{1}(\mathrm{t})+\omega_{0}(\mathrm{t}) \mu_{0}(\mathrm{t})
$$

where $\mu_{i}$ are the average value of background and foreground classes. $\omega_{i}$ are probabilities for the two classes given by the relative number of pixels in each class separated by the threshold $\mathrm{T}$. Then, the gradient information $\mathrm{I}_{G}$ whose gradient levels exceed $\mathrm{T}$ is generated as follows:

$$
\mathrm{I}_{\mathrm{G}}(x, y)=\left\{\begin{array}{cc}
255 & G(x, y) \geq T \\
0 & G(x, y) \prec T
\end{array}\right.
$$


In this study, we use the Vertical Mean Distribution (VDM) method proposed by [13] to identify the horizon line. The following equation determines the VMD is:

$$
V M D(i)=\frac{1}{W} \sum_{j=1}^{W} I_{G}(i, j) ; \in(1, H)
$$

where, $\mathrm{VMD}$ (i) is the average gray of each row. $W$ and $H$ is the width and the height of the original image. $I_{G}(i, j)$ is the intensity of the pixels obtained by Otsu threshold previously used. Fig.2(f) shows an example of horizon line detection using gradient information extarcting by Otsu threshold. The part under the horizon line is defined as the ROI.

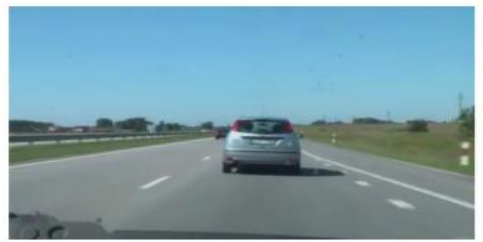

(a) Original image

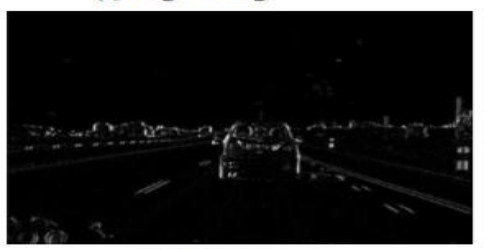

(c)Gradient image

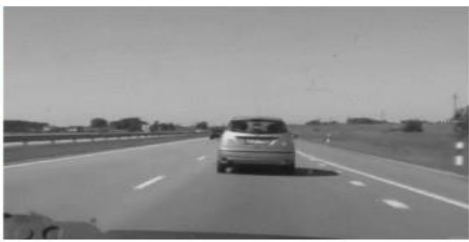

(b) Grayscale image

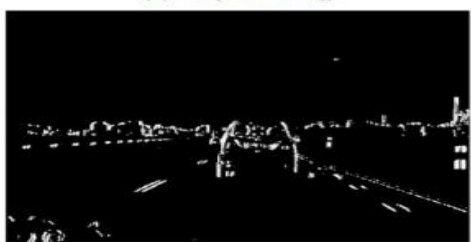

(d) Otsu Threshold

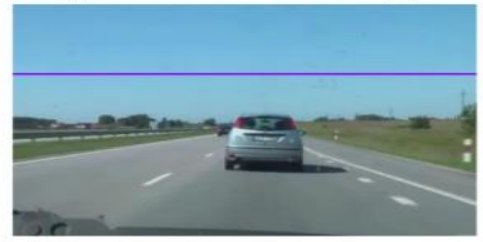

(f) Horizon line and

ROI detection

Fig.2. Shows Flow Chart of Pre-processing Step

\section{- Learning Process}

The learning phase is composed of three parts: preprocessing, extraction of the features vector and training phases. During this process, for each image, after preprocessing (the input color image is converted to the grayscale image), a descriptor HOG is used to extract a feature vectors. Finally, we use the feature vectors selected from the first step for get training base with a Support Vector Machine (SVM) classifier. The support vector machine used for training is a fast and robust classifier. The datasets used in our algorithm proposed contains positive images (vehicle) and negative images (not vehicle) as shown in Fig. 3. 

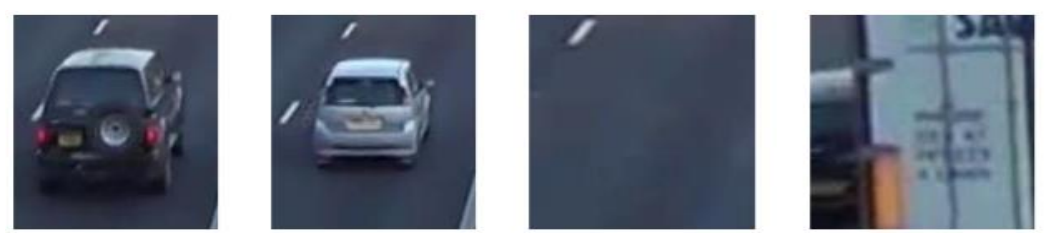

Fig.3. (a) Positive Images; (b) Negative Images

\section{1) Histogram of Oriented Gradients (HOG)}

The HOG descriptor is one of the most popular descriptors used for feature extraction in local region by taking orientation histograms of edge intensity. At first, to obtain the HOG descriptor, the gradient image is calculated by an appropriate filter mask in order to extract the edge gradients and orientations. Next, depending to their gradients and orientations, a grid of histograms is created, where every histogram votes the respective gradients using its length into small spatial area called "cell". To preserve locality, the histograms of edge gradients are computed for each cell. Therefore, the cells themselves are then organized in rectangular blocks (each cell comprises the same number of gradients). So, to form a features vector, the histogram values of all cells in each block are concatenated. The feature vector of each block is normalized and finally, to form the final feature vector, the concatenation of all those block-vectors is necessary.

\section{2) Linear Support Vector Machines Classifier}

The features vectors extracted by any descriptor are classified by linear Support Vector Machines (SVM). SVM is discriminative classifier widely used in different pattern recognition tasks. The SVM classifier may realize separation between classes with an optimal hyperplane. In this study, we use the SVM classifier to calculate an optimal hyperplane that categorizes a set of labels training data in the correct class. The optimal hyperplane is solved as follow:

$$
D=\left\{\left(\mathrm{x}_{\mathrm{i}}, \mathrm{y}_{\mathrm{i}}\right) \mid \mathrm{x}_{\mathrm{i}} \in R^{n}, \mathrm{y}_{\mathrm{i}} \in\{-1,1\}, i=1 \ldots . l\right\}
$$

Given $\mathrm{x}_{\mathrm{i}}$ is the training features vectors and $\mathrm{y}_{\mathrm{i}}$ the corresponding set of $l(1$ or1-) labels. An SVM tries to find a hyperplane to distinguish the samples with the smallest errors. The classification function is obtained by calculating the distance between the input vector and the hyperplane.

$$
w \cdot x-b=C_{f}
$$

where $w$ and $b$ are the parameters of the model.

\subsection{Detection Phase}

The detection process, also, is composed of three parts: preprocessing, extraction of the features vector and detection phases. 


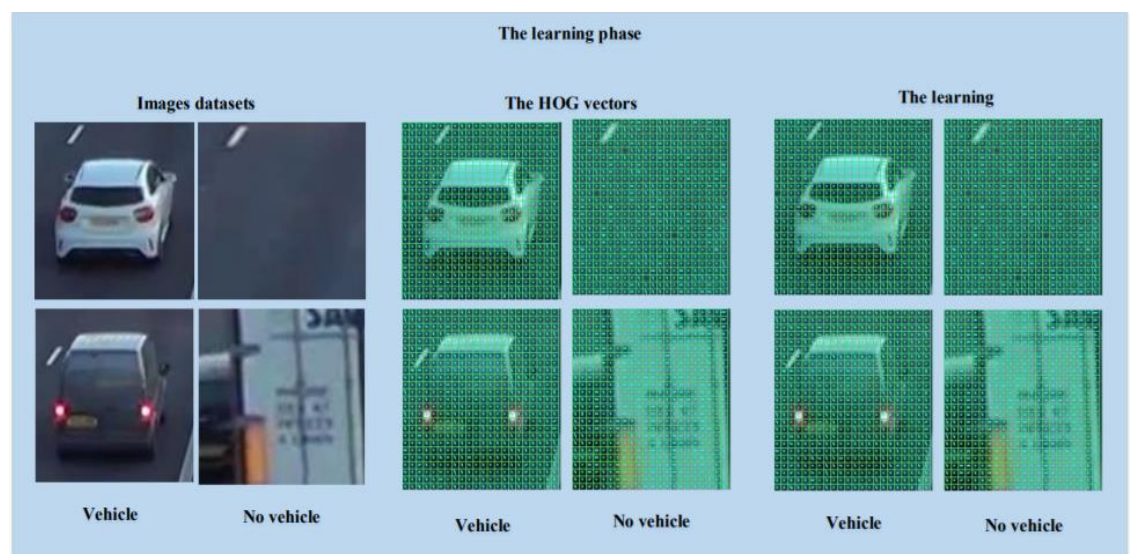

Fig.4. The Learning Process

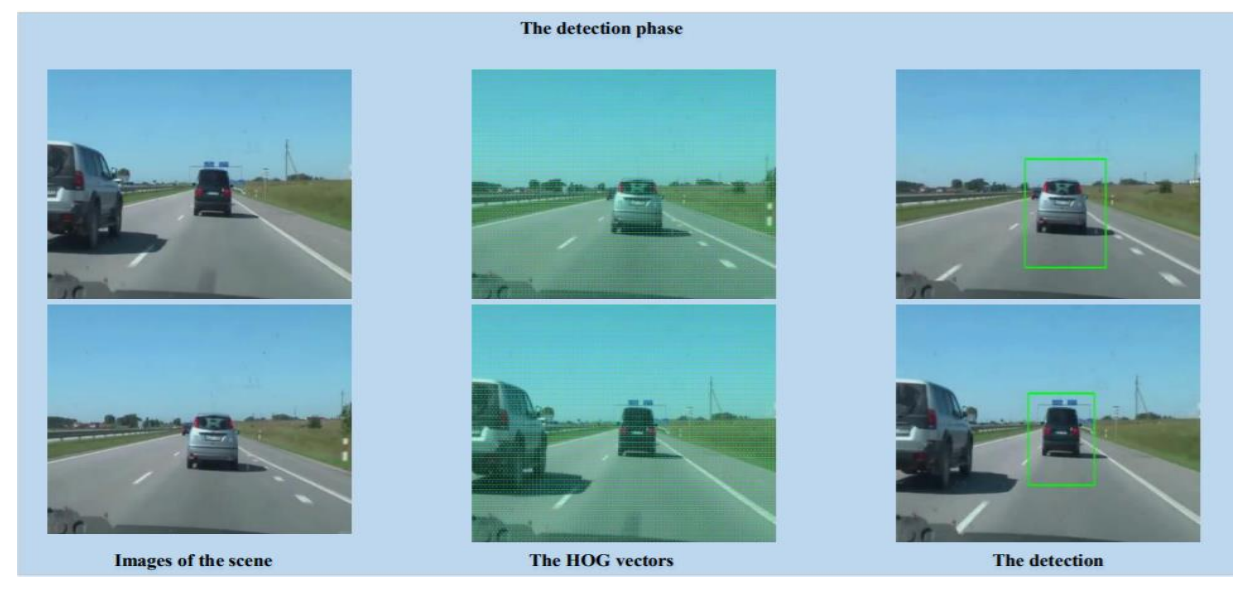

Fig.5. The Detection Process

In the feature extraction phase, the descriptor is used to select the feature vector for each test image. Each test image from sequence video (with vehicles or not) separated in thumbnails, using a sliding window having the same size used in the feature extraction phase for learning process. The descriptor HOG is used to extract a features vector. Finally, we use the training base in learning process with classifier SVM to indicate whether the test image presents a vehicles or not. The Fig.5 shows the features extraction and detection phases for detection process. The Fig. 4 and Fig. 5 are shows the following two processes (learning process and detection process). Experimentally, we found that this vehicles obtained using HOG algorithm and SVM classifier can very probably be vehicles candidates for the lane collision warning step.

\subsection{Lane Collision Warning}

Lane Collision Warning System (LCWS) plays a crucial role in our proposed algorithm. The lane collision is based on the previous cited steps (process detection, which can be used for lane collision step). Lane collisions means a situation when the vehicle is collision from the front vehicle. Once the front vehicle is detected, a lane collision can be calculated based on the distance between the origin of ROI placed at the coordinate $(\mathrm{X} / 2,0)$ and the front vehicle as follows. Let X be the width of the road, D the difference between the vehicle A2 and 
A1, Xc and Yc the two axis in vehicle coordinate as depicted in Fig. 6. The front vehicle position with respect to the road is obtained using equations (9).

$$
D=\sqrt{\left(x_{A 1}-x_{A 2}\right)^{2}+\left(\mathrm{y}_{A 1}-y_{A 2}\right)^{2}}
$$

Let us consider D the distance between A2 and A1, T c is a proper threshold value. However, we used the measure $\mathrm{D}$ to determine the lane collision. If $\mathrm{D}$ is lower than Tc, the vehicle in the normal position. If $\mathrm{D}$ is higher than Tc, the vehicle in the dangerous distance, and a lane collision warning is issued. Finally, we obtain the lane collision detection with its distance as follows:

$$
\text { Collision }=\left\{\begin{array}{l}
\text { normal distance if } D<T c \\
\text { dangerous distance if } D>T c
\end{array}\right.
$$

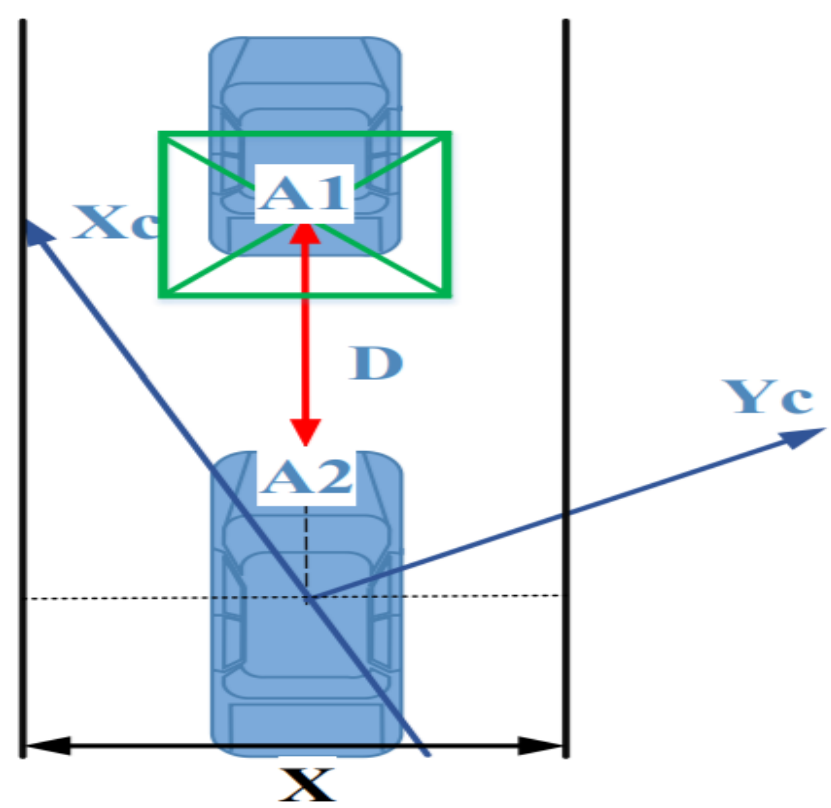

Fig.6. The Relationship between the Vehicle Position and the Front Vehicle Condition.

\section{Experimental Results}

Different dataset in a different environments, and under various lighting conditions were analyzed to assess vision-based Driving Assistance Systems. Given that the performance of our proposed system varies depending on lighting changes, we believe are there various lighting conditions for building the dataset. Artificial light changes and natural light changes can include the lighting changes in road environments. The vehicle lamps and street features can make artificial light changes as well as weather and time that make natural light changes. We tested our algorithm under various conditions involving these last constraints as shown in TABLE 1 . The system is subjected to serious tests including realistic constraints such as pedestrians, obstacles, and traffic by using highways and local city roads datasets. All datasets were captured with a resolution of $640 * 500,640 * 480$, $640 * 360$ pixels. We implemented the proposed methods in $\mathrm{C}++$ and OpenCV. 
Table 1. Different Conditions for Vehicle Detection

\begin{tabular}{lll}
\hline Datasets & Time & Different Conditions \\
\hline Highway Roads & Day, Sunrise, Sunset & Clear/ Rainy /Cloudy \\
Local City Roads & Night & Street lamps/Car lamps \\
Urban Roads & Day & Shadows / Streets \\
\hline
\end{tabular}

In this study, three steps are presented in our system. The first step is done by preprocessing and learning process in order to extract the ROI and get the training base. The features extraction using the HOG descriptor and training with SVM classifier. The second step is done by used the training base for vehicles detection in the road. The final step is done by determines if there is traffic collision with the vehicle ahead or not, depending on the car position. Thus, in order to evaluate our proposed approach (HOG with SVM), we tested our descriptor with SVM classifier for vehicles detection and recognition under various conditions and environments. We compute the detection rate for our method, in order to show the efficient for vehicle detection and recognition, and for real-time testing applied to videos sequences. The detection rate is calculated as follows:

$$
\mathrm{DR}=\frac{\mathrm{C}}{\mathrm{N}} * 100 \%
$$

where $\mathrm{C}$ is the number of correctly detected vehicles, $\mathrm{N}$ is the number of whole vehicles and DR is the detection rate. We randomly sample 573 positive images with vehicle and 1436 negative images without vehicles for training. We use another base (positive/negatives images) for testing. The linear SVM is used to train and classify the images. Then, we display the extraction performance of the HOG descriptor. Their features are all extracted from blocks of the same size $64 * 128$ pixels. Table 2 shows the extraction performance of this descriptor for vehicle detection.

Table 2. Face Recognition Rate of Our Proposed System.

\begin{tabular}{llll}
\hline Method & Total images & Detected vehicles & DR $(\%)$ \\
\hline HOG(positive images) & 286 & 278 & 97.20 \\
HOG(negative images) & 735 & 709 & 96.46 \\
HOG(P/N) & 1021 & 987 & 96.83 \\
\hline
\end{tabular}

Experimental results show satisfactory performance of HOG for vehicles detection with an average detection rate greater than $96.83 \%$ under various conditions, which is better than the Haar descriptor [7] with an average detection rate $91.3 \%$. By using our proposed method, we have to add a few treatment to perform the recognition step. Therefore, by the proposed approach, we use a unified method for learning and detection at the same time, without using separate algorithms for each step. Over than 5000 images from the datasets in different weathers and lighting conditions are used to evaluate the performance of our proposed algorithm for vehicles detection and experimental results are shown in Fig. 7.

Although, our proposed algorithm for vehicles detection shows an excellent efficiency for multiple datasets realized under different lighting conditions and always returns a few false detections as shown in Fig. 8. In the Fig. 7, the green rectangular shown the vehicles detected by our system. The processing time takes $0.02 \mathrm{~s}$ for vehicle detection. This method it is can process robustly applied in real-time to identify most vehicles with complex mark. Our system gives good result in complex situations of the road since it considers only the 
vehicle with the shadow.

One of the critical applications in vehicle detection is the lane collision warning. However, an involuntary lane collision happens in case of driver fatigue, inattention or drowsiness. The latter situation is treated differently by our algorithm. For safety purposes, when an involuntary lane collision happens, an alert message will be sent to the driver. We considered some examples of involuntary lane collision as shown in Fig. 9. These figure indicate that the vehicle in the dangerous distance to the front vehicle. In this work, D is compared to a threshold Tc, and a lane collision warning is issued if D> T c . Experimental results and evaluation show that $\mathrm{T}$ $100 \mathrm{c}=$ is the best choice. The Fig. 9 shows the case where the vehicle is going to lane collision (when an involuntary lane collision is detected), and an alert message in real time is sent to the driver.

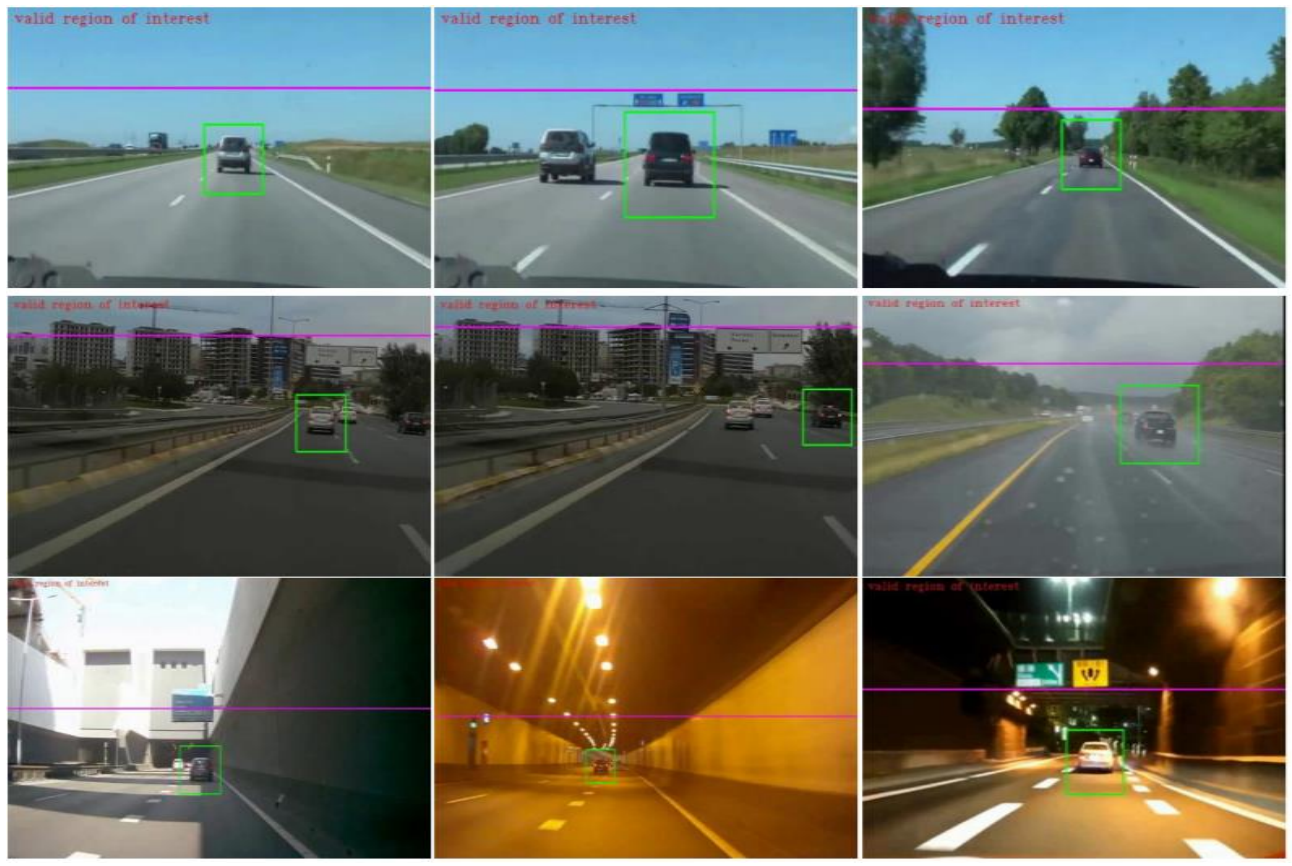

Fig.7. Vehicles Detection Results of the Datasets in Different Lighting Conditions

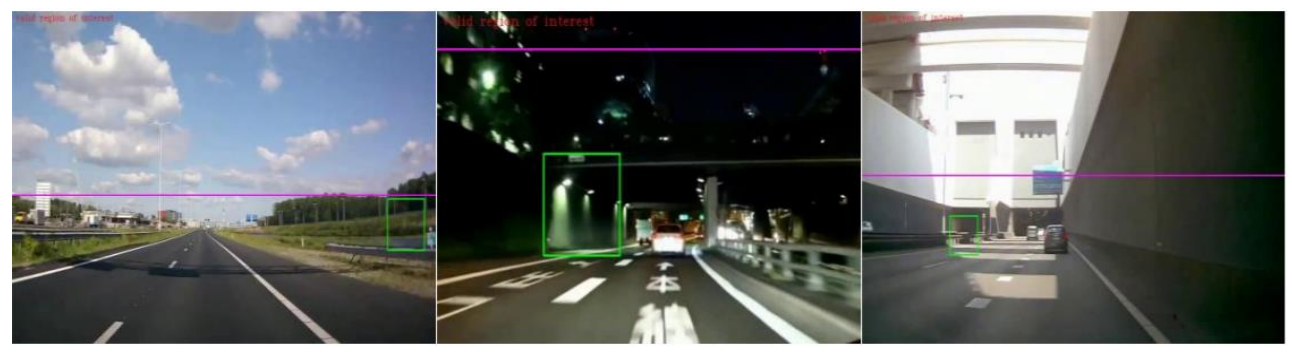

Fig.8. Examples of the False-detection Result 


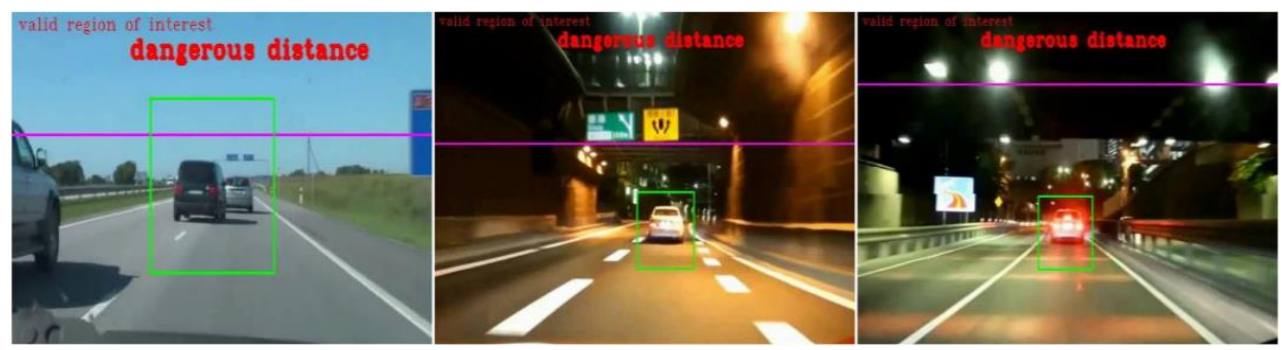

Fig.9. Corresponding Distance D.

In most cases, the correct detection rate averages is greater than $97 \%$ in various lighting conditions. Furthermore, the entire process takes only $48 \mathrm{~ms}$ per frame for the three steps, which makes our proposed algorithm well suitable for real-time vehicle detection systems.

\section{Conclusions}

In this paper, we proposed a novel reliable real-time lighting-invariant lane collision warning system. Initially, horizon line detection was investigated to localize the ROI that decrease the computational complexity. In fact, a combination of the Otsu's threshold method (to deal with lighting problems, enhance the results of pre-processing step and to ensure the ROI detection) and, the descriptor HOG combined with SVM classifier are used to get training base. Then, we introduce the training base to localize the current vehicle in the road. Finally, the distance between the vehicle and the vehicle detected in front is calculated and a decision on the collision of the vehicle is issued in real time. Experimental results indicate the efficiency of our proposed system.

The correct detection rate averages is greater than $97 \%$ in various lighting conditions. Furthermore, the entire process takes only $48 \mathrm{~ms}$ per frame for the three steps, which makes our proposed algorithm well suitable for real-time vehicle detection systems.

In this work, much attention is paid to the lighting problems, which is an important factor for reliable driving assistance systems. The proposed algorithm gives better detection performances under various lighting conditions; however, there are some restrictions in some special situations. In fact, the proposed algorithm does not manage multiple extremely difficult situations. To overcome these limitations, we should establish Kalman filter for tracking vehicles in the road.

\section{References}

[1] HUANG, Shih-Shinh, CHEN, Chung-Jen, HSIAO, Pei-Yung, et al. On-board vision system for lane recognition and frontvehicle detection to enhance driver's awareness. In : Robotics and Automation, 2004. Proceedings. ICRA'04. 2004 IEEE International Conference on. IEEE, 2004. p. 2456-2461.

[2] Z SUN, Zehang, BEBIS, George, et MILLER, Ronald. On-road vehicle detection using Gabor filters and support vector machines. In: Digital Signal Processing, 2002. DSP 2002. 2002 14th International Conference on. IEEE, 2002. p. 1019-1022.

[3] KARADUMAN, O., EREN, H., KURUM, H., et al. Approaching car detection via clustering of vertical-horizontal line scanning optical edge flow. In: Intelligent Transportation Systems (ITSC), 2012 15th International IEEE Conference on. IEEE, 2012. p. 502-507.

[4] XIONG, Bin et DING, Xiaoqing. A generic object detection using a single query image without training. Tsinghua Science and Technology, 2012, vol. 17, no 2, p. 194-201. 
[5] XU, Yanwu, XU, Dong, LIN, Stephen, et al. Detection of sudden pedestrian crossings for driving assistance systems. IEEE Transactions on Systems, Man, and Cybernetics, Part B (Cybernetics), 2012, vol. 42, no 3, p. 729-739.

[6] BARCELLOS, Pablo, GOMES, Vitor, et SCHARCANSKI, Jacob. Shadow detection in camera-based vehicle detection: survey and analysis. Journal of Electronic Imaging, 2016, vol. 25, no 5, p. 051205051205 .

[7] MOHAMED, Atibi, ISSAM, Atouf, MOHAMED, Boussaa, et al. Real-time Detection of Vehicles Using the Haar-like Features and Artificial Neuron Networks. Procedia Computer Science, 2015, vol. 73, p. 24-31.

[8] DALAL, Navneet et TRIGGS, Bill. Histograms of oriented gradients for human detection. In: Computer Vision and Pattern Recognition, 2005. CVPR 2005. IEEE Computer Society Conference on. IEEE, 2005. p. 886-893.

[9] CAI, Yingfeng, SUN, Xiaoqiang, WANG, Hai, et al. Night-Time Vehicle Detection Algorithm Based on Visual Saliency and Deep Learning. Journal of Sensors, 2016, vol. 2016.

[10] YONG, Xi, ZHANG, Liwei, SONG, Zhangjun, et al. Real-time vehicle detection based on Haar features and Pairwise Geometrical Histograms. In: Information and Automation (ICIA), 2011 IEEE International Conference on. IEEE, 2011. p. 390-395.

[11] O. Nobuyuki, "A threshold selection method from gray-level histograms." IEEE Transactions on Systems, Man, and Cybernetics, Vol.9, No. 1, pp. 62-66, 1979.

[12] Nagashree, R. N., and N. Aswini. "Approaches of Buried Object Detection Technology." International Journal of Wireless and Microwave Technologies (IJWMT), (2014).

[13] Aetesam, Hazique, and Itu Snigdh. "A Survey on Topology Maintenance in Wireless Sensor Networks." International Journal of Wireless and Microwave Technologies (IJWMT) 6.4 (2016): 29-37.

[14] Pradhan, Shibashis, Sudipta Chattopadhyay, and Sujatarani Raut. "A novel orthogonal minimum correlation spreading code in CDMA system." IJ Wirless and Microwave Technologies 2 (2014): 38-52.

\section{Authors' Profiles}

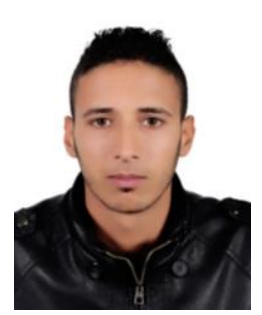

Yassin Kortli received the master of research degree in Microelectronics and Nanoelectonics from Faculty of Sciences of Monastir, University of Monastir in 2015. Since this date he has been PHD degree in Computer vision. His research interests include Hardware/Software co-simulation, pattern recognition, image and video processing, Multiprocessor System on Chip (MPSoC).

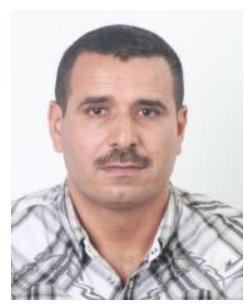

Mehrez Marzougui received the B.Sc. degree in Microelectronics from Tunis University in 1996, the M.Sc. degree and the Doctorate in Microelectronics from Monastir University in 1998 and 2005 respectively. Since this date he has been Assistant professor in Computer engineering. His research interests include Hardware/Software co-simulation, image processing, Multiprocessor System on Chip (MPSoC). 


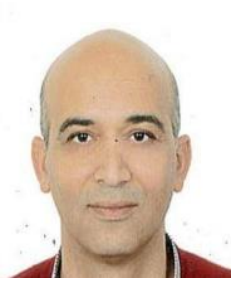

Atri Mohamed born in 1971, he received his PhD Degree in Micro-electronics from Faculty of Science of Monastir, Tunisia, in 2001 and his Habilitation in 2011. He is currently a member of the Laboratory of Electronics \& Micro-electronics. His research includes circuit and system design, pattern recognition, image and video processing.

How to cite this paper: Yassin Kortli, Mehrez Marzougui, Mohamed Atri," High-reliability Vehicle Detection and Lane Collision Warning System", International Journal of Wireless and Microwave Technologies(IJWMT), Vol.8, No.2, pp. 1-14, 2018.DOI: 10.5815/ijwmt.2018.02.01 